\title{
COMPARISON OF AIRFOIL AND RIBBON FAIRINGS FOR SUPPRESSION OF FLOW-INDUCED VIBRATIONS
}

\author{
S. M. FANG \& J. M. NIEDZWECKI \\ Zachry Department of Civil Engineering, Texas A\&M University, College Station, Texas, USA.
}

\begin{abstract}
Cables and various subsea product flow-lines are often subjected to flow-induced vibrations as a consequence of wave or wave and current flow excitation. An experimental study that explores the comparative suppression effectiveness of airfoil and ribbon fairings is presented. In the experimental investigation, an instrumented tensioned $29 \mathrm{~m}$ horizontal composite cylinder with a length to diameter ratio of 760 is subjected to a range of uniform current and combined current and regular wave conditions. In the analysis of the cross-flow response amplitude ratios, modal parameters, such as mode shape, damping ratio were extracted. The analytical procedures were performed using a modified time domain decomposition technique. The analysis illustrated the amplified response behavior for bare cylinders, and illustrated that full coverage by airfoil and ribbon fairings could effectively suppress flow-induced vibrations in current only conditions, but both fairings were ineffective when regular wave conditions were superimposed on the uniform current conditions, even though the airfoil provided larger damping than ribbon fairings. Under combined wave and current conditions, the response behavior of either suppression device was found to be insensitive to coverage densities.

Keywords: Airfoil fairing, combined loads of current and waves, coverage density, flow-induced vibration, ribbon fairing, Time domain decomposition, vortex-induced vibration.
\end{abstract}

\section{INTRODUCTION}

Subsea cables, free-spanning pipelines and a variety of slender structural components are often subjected to current and wave conditions that may lead to damage due to vortex-induced vibrations (VIV). Nielsen, Soreide and Kvarme [1] reported on a free-spanning pipeline designed for the Ormen Lange field, located off the western coast of Norway, which had a length to diameter ratio (L/D) of 846 , which is much larger than the typical limit value of 120, proposed by Det Norske Veritas [2]. The complexity of offshore installations involving combined environmental loads on large-scale structures supports the need of experimental study to investigate the system response behavior and the potential requirement for VIV suppression in certain design conditions.

A brief summary of some VIV experiments studying the response of horizontally mounted cylinders is presented in Table 1. The studies presented can logically be grouped into three categories based upon their L/D ratio and the type of VIV suppression device used in the tests. The first group focused on the VIV suppression performance for small L/D ratios $(<30)$, where such experimental setup facilitated testing devices with multiple geometries in high Reynolds number flow [3, 4]. Allen, Henning and Lee [3] tested five different airfoil fairings with chord to thickness ratio (c/t) values ranging from 1 to 2 at super-critical flow regime. It was determined that an airfoil fairing with chord to thickness ratio (c/t) of 1.18 was overall the best choice for it was both cost effective and efficient in reducing the root-mean-squared (RMS) cross-flow responses from $0.43 \mathrm{D}$ to $0.13 \mathrm{D}$. The second group that focused on experiments studied VIV for flexible cylinders with intermediate or large L/D ratios. And these research studies were especially focused on multi-modal motions, the effect of pretension and the coupling effect between in-line and cross-flow vibrations. The experimental studies reported by $\mathrm{Li}$ et al. [5], Prastianto, Otsuka and Ikeda [6] and Wilde and Huijsmans [7] are included in Table 1. 


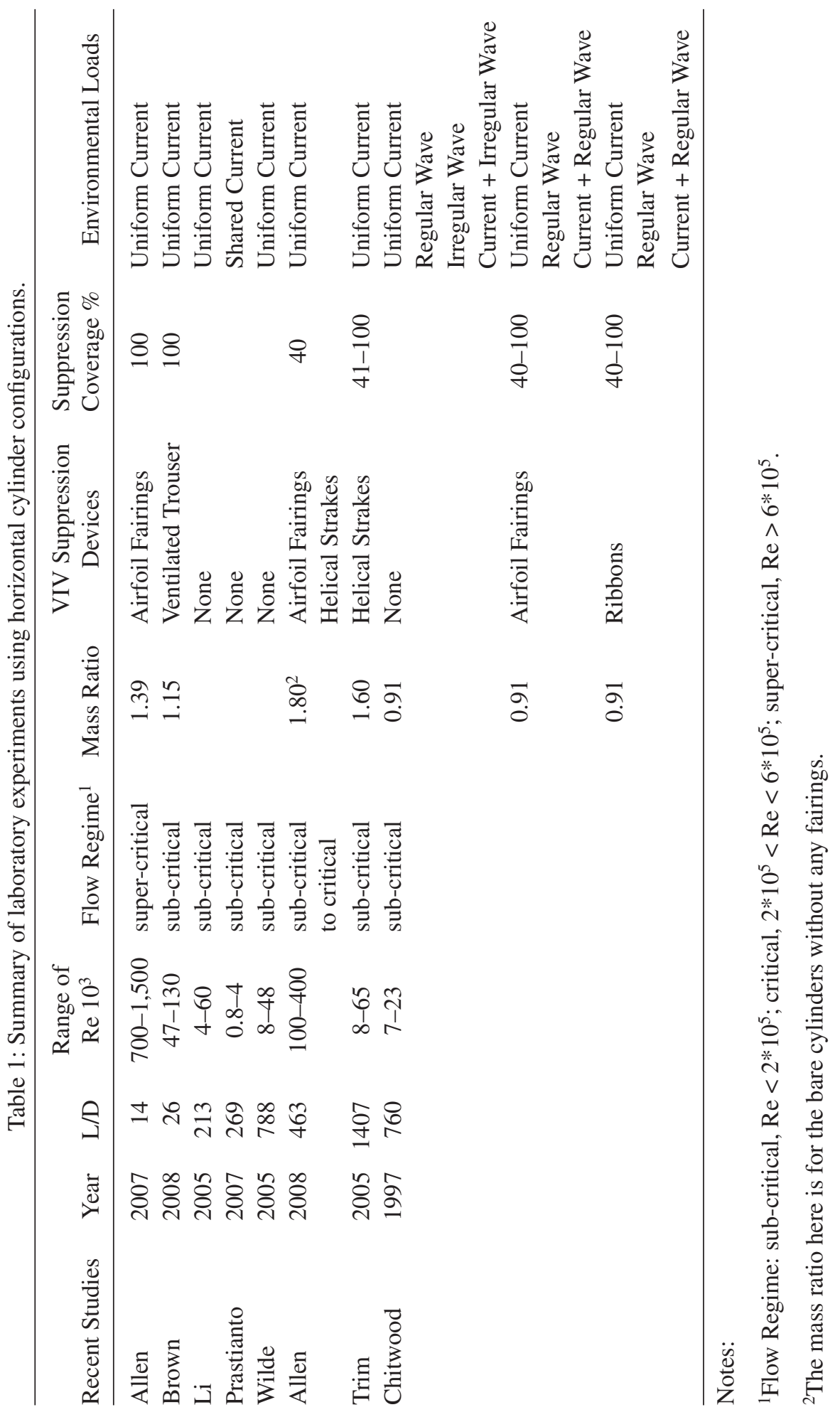


In these studies, the L/D ratios were 213, 269 and 788 respectively, and each of those experiments were performed in the sub-critical flow regime. Li et al. tested a bare cylinder for 27 different current velocities ranging from 0.1 to $1.5 \mathrm{~m} / \mathrm{s}$ with pretensions of $200 \mathrm{~N}$ and $600 \mathrm{~N}$, however no frequency lock-in or resonance was observed. Prastianto et al. reported an experiment at a relatively low uniform current velocity of $0.2 \mathrm{~m} / \mathrm{s}$, at which both the $3 \mathrm{rd}$ and 9 th mode were excited with the lower mode being dominant. Similarly, Wilde and Huijsmans showed that in a test with current velocity of $1.0 \mathrm{~m} / \mathrm{s}$ and pretension of $900 \mathrm{~N}$, the 5th or 6th mode was dominant and the 11th or 12th mode participated. As studies by group two demonstrated, multi-modal responses were frequently observed in VIV for these L/D ratios and both current velocities and pretensions affected the dominant modes. Strong coupling effects between in-line and cross-flow responses were also observed in these studies.

The third grouping of research studies focuses on the performance of various VIV suppression devices on long flexible cylinders. Allen and Henning [8] conducted experiments using a cylinder with an L/D ratio of 463 in sub-critical to critical flow regime, and compared the performance of helical strakes (17.5D pitch, 0.25D height) and short fairings (c/t ratio 1.5). Both devices were in $40 \%$ partial coverage and deployed from one end. The results revealed strakes and fairings can both effectively suppress the responses in the covered end, but that airfoil fairings outperformed strakes by providing more damping overall to the system. Trim et al. [9] compared helical strakes with 2 geometries, 17.5D pitch, 0.25D height versus 5D pitch, 0.14D height. The cylinder with an L/D ratio of 1407 was towed in sub-critical flow regime. The experiments demonstrated that the first strake design was more effective in mitigating VIV than the second, and reduced the estimated fatigue damage by one-order-of-magnitude. Coverage of $91 \%, 82 \%, 62 \%$ and $41 \%$ of strakes were studied and the results clearly showed that strake performance declined rapidly when coverage was smaller than $82 \%$.

The experimental results reported here fit with the third grouping of research studies and expand upon the initial research reported on ribbon fairings by Niedzwecki and Fang [10]. The study provides a comparison of airfoil fairings and ribbon fairings with both complete and partial coverage. Ribbon fairings are generally used to suppress VIV on cables, but have shown some advantages over airfoil fairings when applied to very flexible systems [11]. Tests comparing the response behavior of the two fairing types were conducted for a range of various uniform current conditions, and combined loads of uniform current and regular waves were also investigated. The multi-modal response behavior of the various test configurations was analyzed using the VIV analysis program developed by Niedzwecki and Fang [10] that incorporates the time domain decomposition technique [12] and the needed practical considerations to recover modal response envelopes and modal parameters.

\section{EXPERIMENT PARTICULARS}

The experiments were conducted with the purpose of investigating the performance of airfoil and ribbon fairings in varying coverage subjected to uniform current or combined loads of current and regular waves. Figure 1 provides an illustration of the test configuration used for the experiments as reported by Chitwood [13]. The composite cylinder used in the tests was $29-\mathrm{m}$ long, with an outside diameter of $38 \mathrm{~mm}$ and an inside diameter of $22 \mathrm{~mm}$. It was estimated to have a unit weight of $10.18 \mathrm{~N} / \mathrm{m}$, and submerged $0.61 \mathrm{~m}$ below the mean water surface. Thus, the composite test cylinder was very light with a mass ratio of 0.91 and quite flexible with an L/D ratio of 760 . As shown in the figure, three wave probes were suspended from the bridge to measure the wave properties, and six bi-axial accelerometers were located 


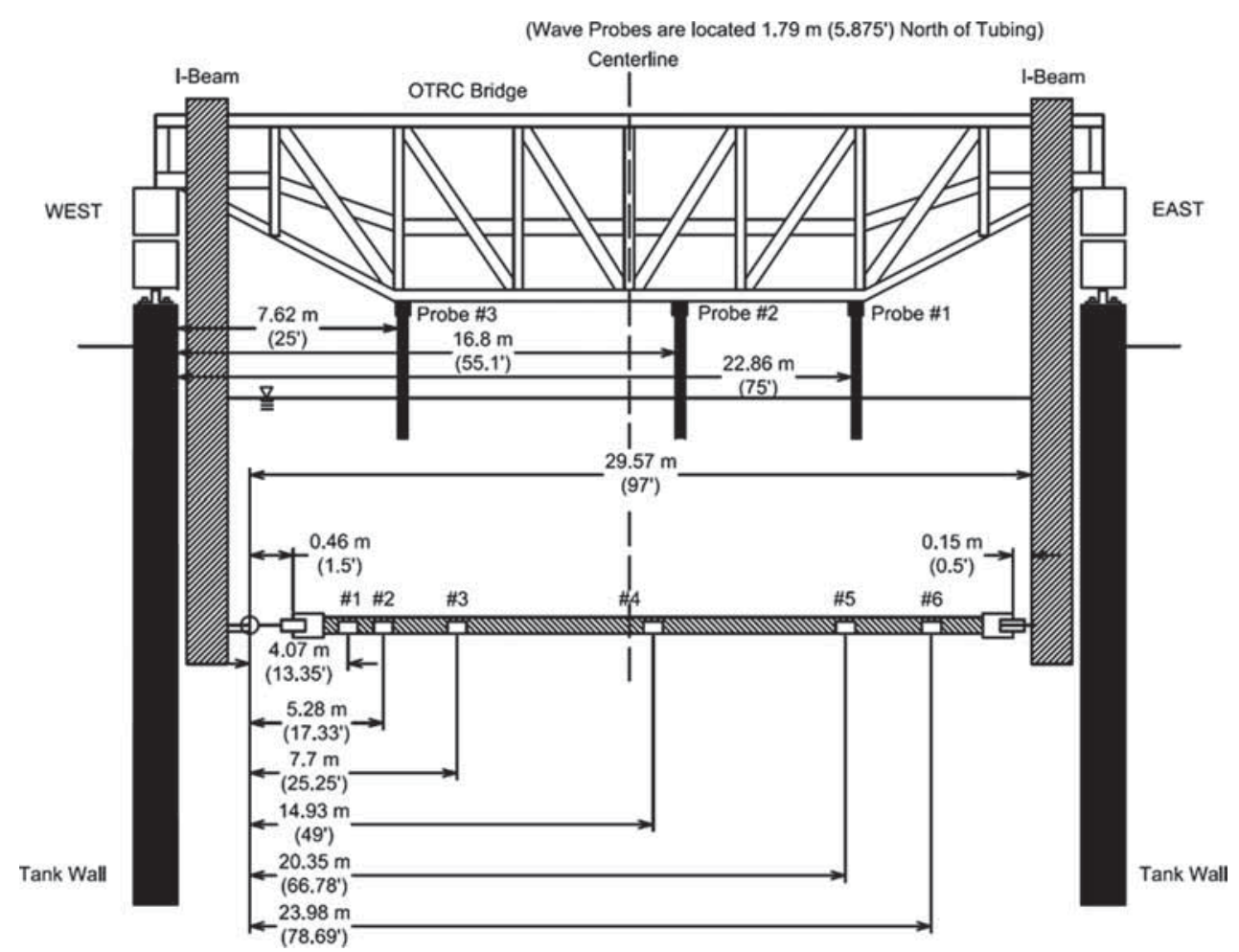

Figure 1: Schematic of the model and instrumentation locations.

at unequal spacing internal to the cylinder. Special care was taken to orient and seal the sensors inside the composite cylinder. Displacement time series were obtained from the recorded acceleration time series through a double integration procedure and the results were compared with the mid-span displacement measured using a string-potentiometer sensor. The bi-axial acceleration measurements enabled estimation of both cross-flow and in-line displacements.

The towing speeds varied between 0.18 and $0.43 \mathrm{~m} / \mathrm{s}$ and simulated uniform current conditions. For the combined uniform current and wave conditions tested, regular waves with the wave height $0.24 \mathrm{~m}$ and the peak period $3.2 \mathrm{~s}$ were introduced. Considering the maximum horizontal wave kinematic velocity, the Reynolds number was estimated to vary between $7 * 10^{3}$ and $2.3 * 10^{4}$, corresponding to sub-critical flow regime.

Photographs in Fig. 2 present side-by-side photos of the airfoil and ribbon fairings used in the study. The airfoil fairings were made of Acrylonitrile Butadiene Styrene plastic (ABS), with a width of $304.8 \mathrm{~mm}(1 \mathrm{ft})$, a chord length of $165.1 \mathrm{~mm}(6.5 \mathrm{in})$ and a thickness of 44.5 $\mathrm{mm}$ (1.75 in) resulting in a c/t ratio of 3.7. The airfoil fairings were attached to the cylinder with zip-ties to ensure free rotation. The ribbon fairings were made of soft compliant vinyl, with the same width as the airfoil fairings, and trailing length of $127 \mathrm{~mm}$ (5 in). The ribbon fairings were secured around the cylinder by zippers on the leading edge. The unit weight of the airfoil and ribbon fairings in air was $5.925 \mathrm{~N} / \mathrm{m}$ and $1.824 \mathrm{~N} / \mathrm{m}$. Note that since the airfoil fairings were not watertight the water trapped inside needs to be counted when considering the total mass. The unit weight for the trapped water was estimated to be $21.680 \mathrm{~N} / \mathrm{m}$. 


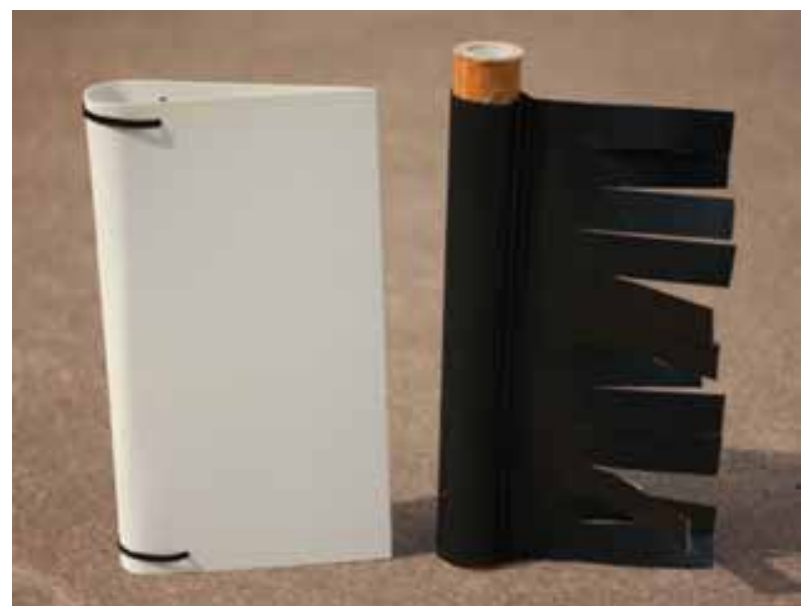

Figure 2: Photographs of the airfoil (left) and ribbon (right) fairings used in the experiments.

The airfoil fairings were tested at 100\%-40\% coverage in $10 \%$ decrements. The fairings were removed from the left end (accelerometer 6). The ribbon fairings were studied at the same coverage range but the reduction from $100 \%$ was done in $20 \%$ decrements.

\section{DATA ANALYSIS USING TIME DOMAIN DECOMPOSITION}

The analysis of the time series data from the towed composite cylinder experiments required a rethinking of the time domain decomposition technique implementation to address subtleties of the phenomena and recorded data. Consequently, the analysis of the data was divided into several interrelated well-defined tasks. First, the cross-flow and in-line displacements were obtained through double integration of the acceleration time series data measured using the bi-axial accelerometers. In addition, the statistical characteristics that included the maximum, minimum and standard deviations of the displacements, measured tension, and current and wave data were obtained. The next major task was to perform a modified time domain decomposition of the displacement time series to recover information on the dominant modes that included the natural frequency, mode shape, modal contribution factor and modal damping.

In evaluation of multi-modal vortex-induced vibration behavior one needs to be careful as the various modes can have the same lock-in range, and the mode of interest might be blended in with other possible modes and might not be correctly recognized. Chung [14] analyzed experimental data from a variety of sources and demonstrated the lock-in range could vary significantly depending upon the mass ratio, in particular cylinders with low mass ratios had a much broader lock-in range than those with higher mass ratios. Figure 3 presents data from this study illustrating the mode number versus the reduced velocity, which is defined here as the ratio of the current velocity to the product of the cylinder's natural frequency times its diameter. The figure presents information for the bare cylinder subjected to three different uniform current velocities $(0.18,0.27$ and $0.43 \mathrm{~m} / \mathrm{s})$. The data suggests that as the uniform current velocities increase so that the corresponding modes 1-3, 2-4, 3-7 might be excited. This type of figure provides some guidance for the various configuration scenarios for the evaluation procedures regarding the expected mode behavior in this study. 


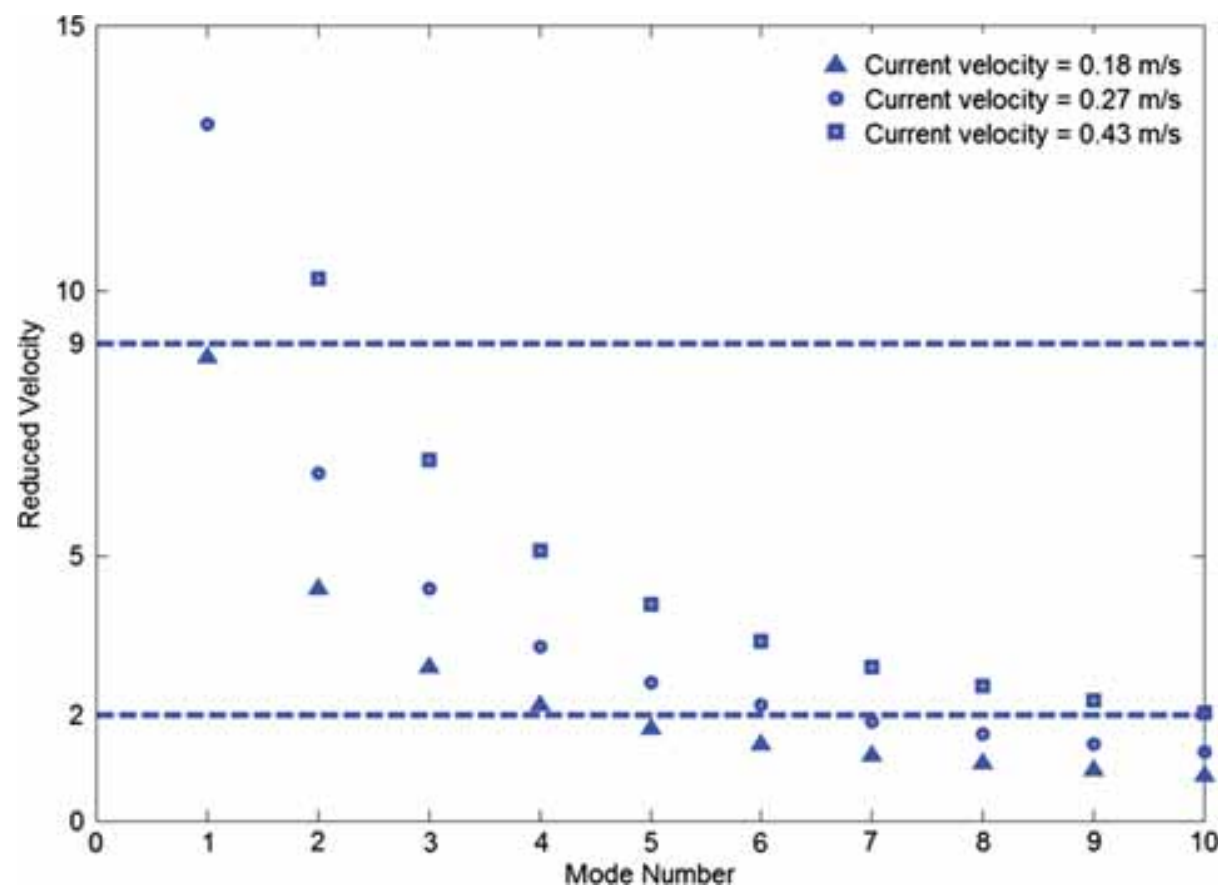

Figure 3: Mode number versus reduced velocity at varied current velocities for bare cylinder.

The key aspects of the time domain decomposition technique include selective isolation of data from time histories, extraction of a certain mode shape from constructed energy matrix using singular value decomposition technique, and calculation of mode contribution factors, damping ratio and other modal parameters for each mode. In order to point out some of the subtleties and modifications associated with the implementation of the time domain decomposition technique for offshore structure applications, the important equations from the original formulation by Kim, Stubbs and Park [12] are included in the discussion for clarity. So beginning with a simple one-dimensional array of $P$ unequally spaced sensors, the acceleration time history of vibrations in cross-flow direction can be assumed as the summation of infinite modes

$$
[\ddot{Y}(t)]=\sum_{i=1}^{\infty}[\ddot{c}(i, t)] * \varphi(i)
$$

where, $\ddot{Y}(t)$ is the cross-flow acceleration and $\ddot{c}(i, t)$ and $j(i)$ are the modal contribution factor and the mode shape at the $i$ th mode respectively. If $N$ samples are recorded, $\ddot{Y}(t)$ is a $p \times N$ matrix. Accordingly for $P$ sensors one can only extract up to the $p^{\text {th }}$ modal parameters without aliasing. The first equation can then be expressed as

$$
[\ddot{Y}(t)]=\sum_{i=1}^{p}[\ddot{c}(i, t)] * \varphi(i)+\varepsilon_{1}(t)
$$

where, $\varepsilon_{1}(t)=\sum_{i=p}^{\infty}[\ddot{c}(i, t)] * \varphi(i)$, denotes a truncation error due to higher modes. 
Spectral analysis and filter theory are applied to isolate the signal of the $i$ th mode $\ddot{Y}(i, t)$ from the array signals. The power spectral densities at all sensors are generated and overlapped to find the energy concentration bandwidth for the targeted mode. Digital filters are designed and used to create a mode-isolated discrete-time response within the appropriate bandwidth. In this analysis a 20th order Butterworth band-pass filter was selected, but for implementation one can choose the optimal filter for the mode isolation process. Utilizing the orthogonality between components of the signal in modal space and the noise space, an energy matrix can be formulated as

$$
[E(i)]=[\ddot{Y}(i, t)] *[\ddot{Y}(i, t)]^{T}=U \Omega U^{T}
$$

where,

$$
\begin{aligned}
& U=[\varphi(i), \psi(1), \psi(2), \ldots, \psi(p-1)] \\
& \Omega=\left[\begin{array}{lllll}
s(i) & & & & \\
& \sigma(1) & & & \\
& & \sigma(2) & & \\
& & \ldots & \\
& & & & \sigma(p-1)
\end{array}\right]
\end{aligned}
$$

The singular value decomposition technique (SVD) is then utilized to decompose the constructed energy matrix, and from eqn (5), the mode shape can be deduced from the first column of $U$. The modal contribution factor with the mode shape obtained previously can be evaluated from the following equation:

$$
[\ddot{c}(i, t)]=\frac{1}{\varphi(i)^{T} * \varphi(i)} * \varphi(i)^{T} *[\ddot{Y}(i, t)]
$$

For the targeted mode, the modal value of the critical damping ratio can be estimated using the Half-Power Bandwidth method applied to eqn (7). Bendat and Piersol [15] in their discussion of the Half-Power Bandwidth method expressed that to obtain accurate estimates, the power spectral density resolution $B_{e}$ requires to be much smaller than the half-power point bandwidth $B_{i}$, specifically $B_{e}<0.2 B_{i}$. When the power spectral density resolution is quite coarse due to the short data lengths, as was the case of the data analyzed in this study, a non-destructive zoom technique can be implemented [16]. This technique is specifically designed to increase the power spectral density resolution near targeted modes by 'zooming in' on the interested frequency range and concentrating all the spectral lines into a narrower band. Consider for example the case of $100 \%$ ribbon faired coverage of the test cylinder in regular waves with a current speed of $0.38 \mathrm{~m} / \mathrm{s}$. For this case the $B_{e}$ resolution was $0.039 \mathrm{~Hz}$ while $B_{i}$ for the first mode was $0.032 \mathrm{~Hz}$, yielding $B_{e}=1.24 B_{i}$ which clearly did not meet the criteria. Applying non-destructive zoom technique, the PSD resolution was reduced to $0.0098 \mathrm{~Hz}$, resulting in $B_{e}=0.2 B_{i}$ and a more accurate estimation of the critical damping ratio was achieved.

As part of the software implementation, a wave-induced vibration simulation model was incorporated in order to estimate the role of the regular wave excitation in the total responses of the bare cylinder configuration, which was subjected to combined uniform current and 
wave loading conditions. The Morison wave force model was adopted to simulate the wave force excitation with a linearized drag term. The derivation of the modal solution is based on results of Niedzwecki and Liagre [17], where string behavior of the model is assumed consistent with the large L/D ratio. The dynamic tension variation was assumed to be negligible to the pretension of the test cylinder.

\section{NUMERICAL RESULTS AND DISCUSSION}

\subsection{Base case of bare cylinder tests}

Prior to the introduction of any fairings, bare cylinder tests were conducted first for a uniform current speed of $0.38 \mathrm{~m} / \mathrm{s}$, and then for the same current speed with the addition of regular waves with $0.24 \mathrm{~m}$ wave height and $3.2 \mathrm{~s}$ period. The normalized maximum and minimum cross-flow displacement envelopes for the cylinder under these conditions are presented in Fig. 4. Upon comparison of the envelopes it is quite evident that regular waves significantly amplify and dominate the bare cylinder response behavior. The targeted 3rd mode vibration behavior can be recognized for both environmental loading conditions. The results also indicate one possibility: the total responses in combined current and wave loads are composed of two components, a fundamental mode dominated by regular waves and a 3rd mode from the vortex shedding forces mainly due to current. Both components of the responses are significant for the bare cylinder cases.

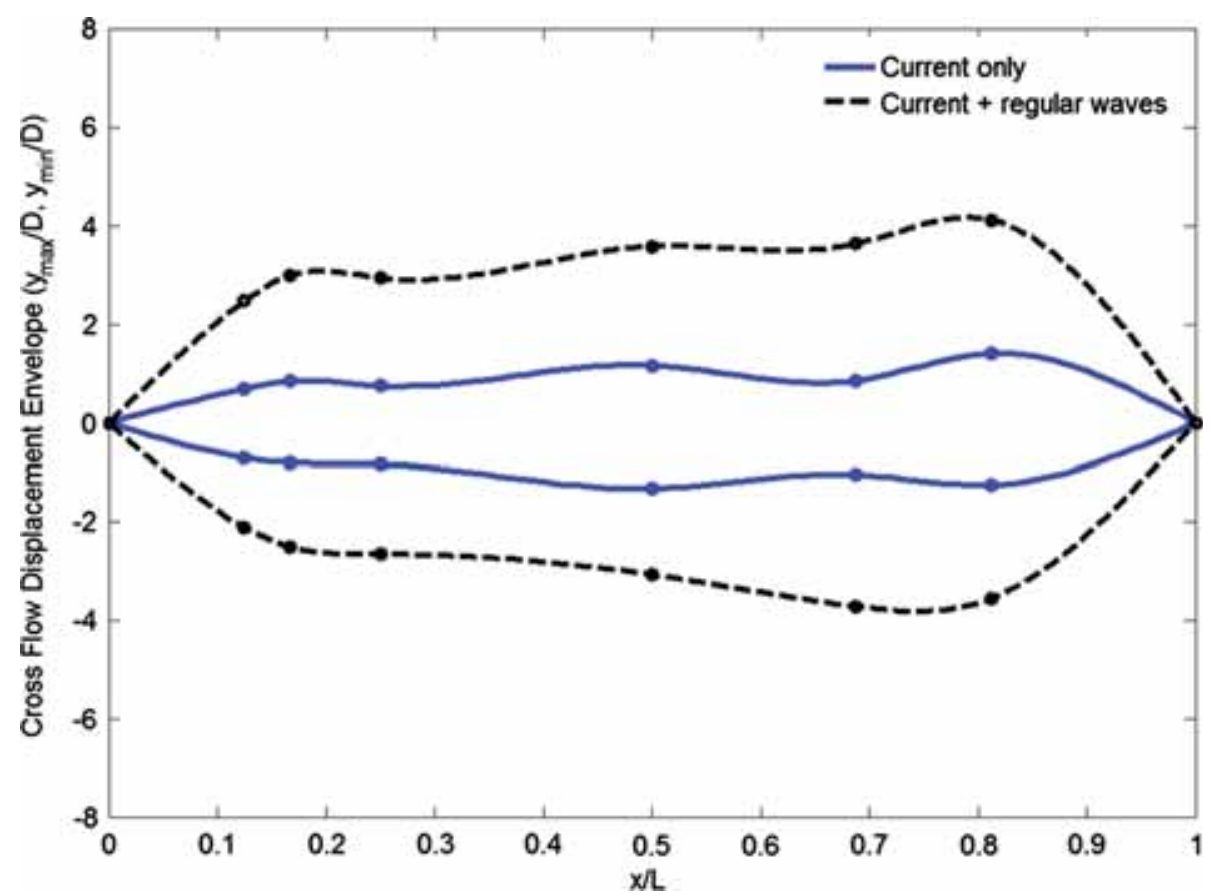

Figure 4: Bare cylinder response in current only $(0.38 \mathrm{~m} / \mathrm{s})$ and combined current $(0.38 \mathrm{~m} / \mathrm{s})$ and waves ( $0.24 \mathrm{~m}$ wave height, $3.2 \mathrm{~s}$ wave period) conditions. 


\subsection{Response behavior with complete airfoil and ribbon fairing coverage}

The next cases of interest involve the comparison of the cylinder with full coverage of airfoil or ribbon fairings. For the bare cylinder tested at current velocities of $0.18,0.24,0.38$ and $0.43 \mathrm{~m} / \mathrm{s}$, the root-mean-square (rms) amplitude ratios at mid-span of the cylinder were measured as $0.12 \mathrm{D}, 0.19 \mathrm{D}, 0.23 \mathrm{D}$ and $0.71 \mathrm{D}$, respectively. For the cylinder test runs with complete coverage by the airfoil fairings, the reduction of the corresponding ratios was found to be $0.04 \mathrm{D}, 0.08 \mathrm{D}, 0.12 \mathrm{D}$ and $0.13 \mathrm{D}$. By way of comparison, for complete coverage of the test cylinder by ribbon fairings the ratios were further reduced over the range of the two middle current speeds. Specifically, the corresponding rms amplitude ratios were determined to be $0.05 \mathrm{D}, 0.06 \mathrm{D}, 0.07 \mathrm{D}$ and $0.13 \mathrm{D}$. Based upon the measured data available the results suggest that the rms responses of the cylinder at mid-span increase with current velocity, and that both airfoil and ribbon fairings significantly suppress the vibrations in the uniform current cases tested.

An example of the cross-flow response behavior for the cases of complete coverage by either airfoil or ribbon fairings when the regular waves were introduced is presented in Fig. 5. For comparison the corresponding bare cylinder case is included in the figure. Clearly the wave kinematics dominated the response behavior and neither fairing type was able to suppress motions. In fact the cylinder equipped with airfoil fairings significantly amplified the vibrations and the one with ribbon fairings somehow kept the responses in similar scale or slightly increased the responses compared with the bare cylinder. The airfoil fairings although free to rotate seemed to accentuate the motion as they followed the wave kinematic motion

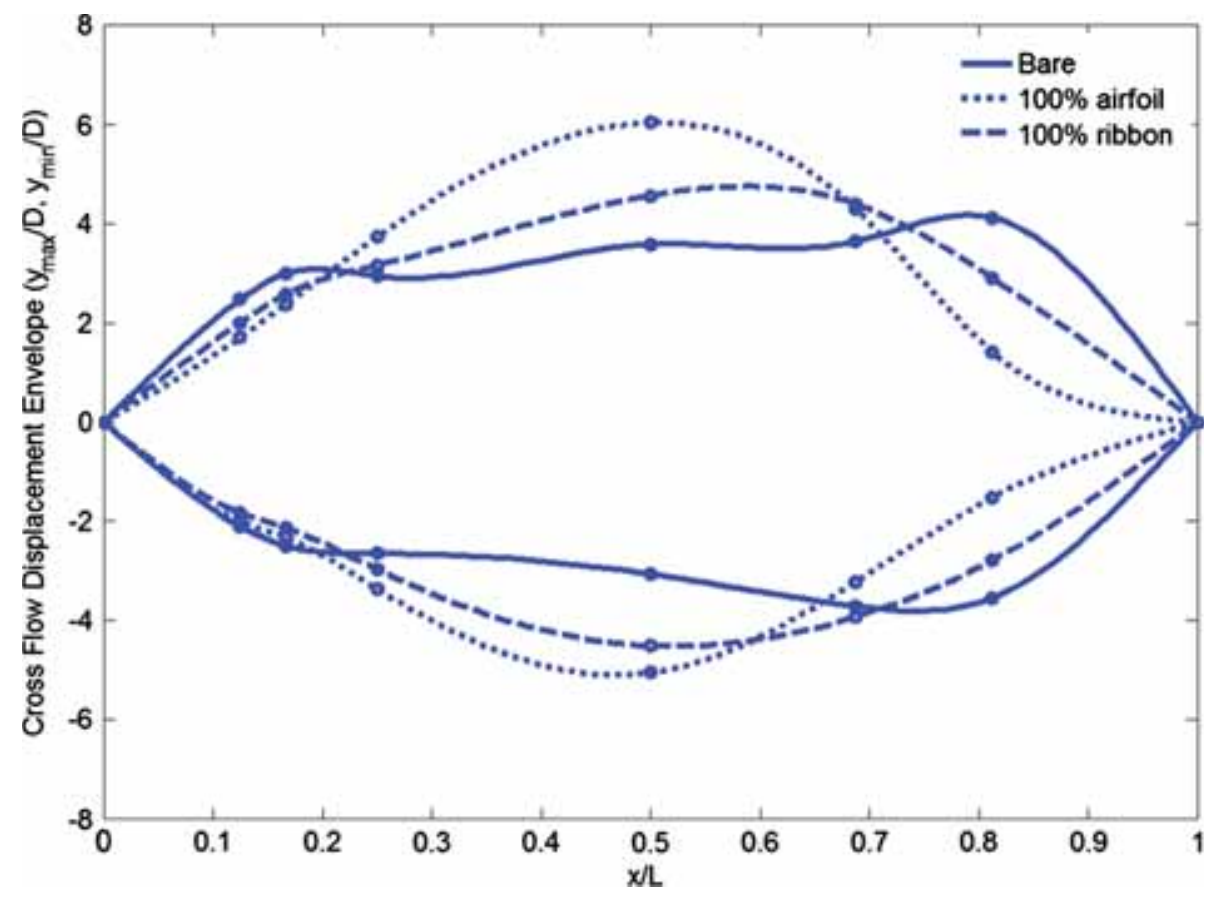

Figure 5: Bare, full airfoil and ribbon faired cylinder response behavior in combined current and waves. 
resulting in increased maximum and minimum rms values especially at the mid-span of the test cylinder. The ribbon fairings were completely compliant and seemed to also respond to the fundamental mode induced by the wave motion, although one could observe the influence of an additional mode in the envelopes. The bare cylinder surface was not completely smooth as the surface reflected the construction of the composite material and had minor indentions and protrusions. Though the fairings tested were originally designed to mitigate flow-induced vibrations in current only situations, the results in Fig. 5 indicate the significantly reduced functionality of both fairings when regular waves were present.

The amplification effect of airfoil and ribbon fairings can be partially explained by comparing the wave peak period and the natural period of the system. For a slightly damped system, the closer the excitation peak frequency approaches one of the natural periods within certain range, the larger the response will be. Considering structure mass, added mass and the mass of possibly trapped water especially for airfoil fairings, the natural periods of the 1st mode for full airfoil, ribbon faired and bare cylinders are estimated to be $2.8,1.9$ and $1.8 \mathrm{~s}$, respectively. Since the wave peak period in the current study is $3.2 \mathrm{~s}$, airfoil faired cylinder has the closest natural period to the waves, followed by ribbon faired and bare cylinder. The rank of this closeness matches well with the actual response envelopes. The amplification effect is also verified by the wave-induced vibration simulated results. Assuming the cylinders with and without fairings are uniform, the wave-induced response amplitude ratios at mid-span were estimated to be 4.8D, 3.5D and 2.0D for full airfoil, ribbon faired and bare cylinders. By way of comparison the measured cross-flow total responses ratios were $5.5 \mathrm{D}, 4.5 \mathrm{D}$ and $3.3 \mathrm{D}$ as shown in Fig. 5, confirming the dominance of the wave kinematics in the observed response behavior.

The power spectral densities for the three cylinder configurations are presented in Fig. 6. The bare cylinder is subject to energy over a large range of frequencies and the effectiveness of the both fairings in reducing this to a few frequencies can be observed. The first peak for each case is centered around the wave frequency with the sequence of peak values

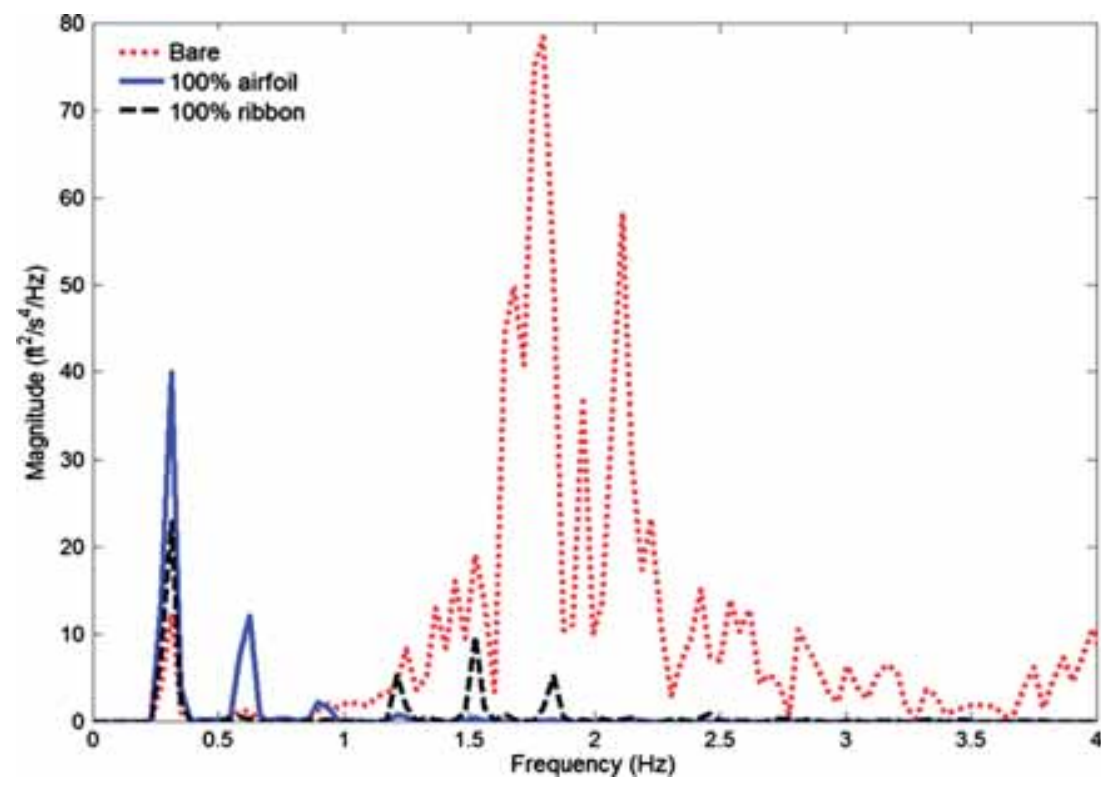

Figure 6: Power spectral densities of bare, fully airfoil and fully ribbon faired cylinders. 
corresponding to the airfoil followed in reduced amplitudes of the ribbon fairing and bare cylinder cases. The few remaining higher frequencies clearly influenced the ribbon fairings envelope when compared with the airfoil fairings, which was even more effective in reducing the effects of higher frequency contributions.

The first three dominant mode shapes of airfoil and ribbon fairings, extracted from measured data using the time domain decomposition technique are presented in Fig. 7a and b, and

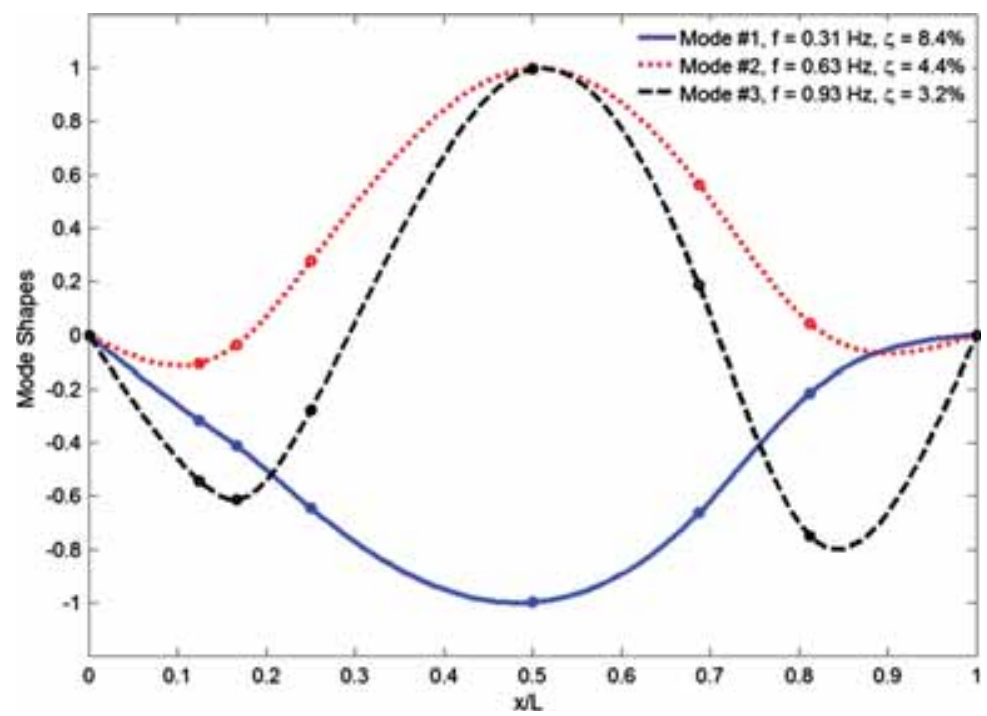

(a)

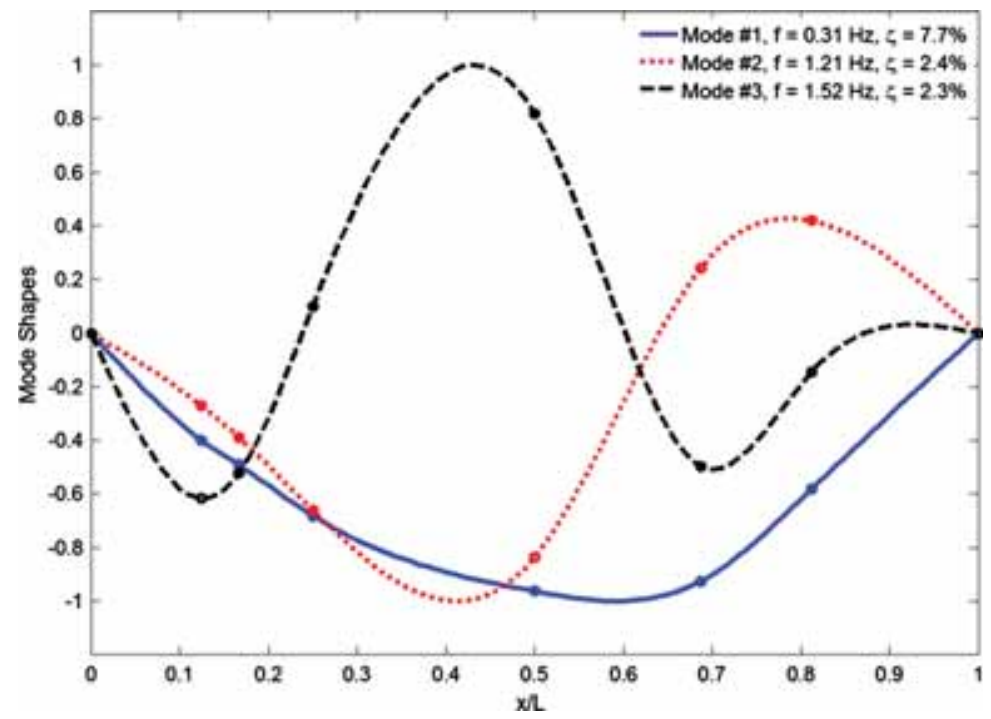

(b)

Figure 7: (a) The three dominant mode shapes for fully airfoil faired cylinder coverage.

(b) The three dominant mode shapes for fully ribbon faired cylinder coverage. 
further validate the noted observations. In particular, the mode shapes corresponding to airfoil fairing coverage appear to be strongly controlled by the fundamental mode, and meanwhile, the multiple mode shapes for the ribbon fairings are explicit and clear, corresponding to the 1st, 2nd and 3rd mode. This is another indicator that in ribbon faired cases, waves were not as dominant as that in airfoil cases, and vortex shedding forces were still significant to excite response in higher mode numbers. The damping ratios of the first three modes using non-destructive zoom technique and Half-Power Bandwidth method were estimated to be $8.4 \%, 4.4 \%$ and $3.2 \%$ for complete coverage by the airfoil fairings, and $7.7 \%, 2.4 \%, 2.3 \%$ for the comparable ribbon fairing case. These results illustrate that the airfoil fairings provide larger damping for the system when compared with the ribbon fairings.

\subsection{Response behavior with partial airfoil and ribbon fairing coverage}

There was a range of coverage configurations as noted in the discussion of the experiment particulars. A comparison of $40 \%$ and $100 \%$ coverage was selected as being representative and is presented in Fig. 8. The results suggest in the combined loading condition, the response magnitudes are not sensitive to coverage, i.e. neither of the suppression devices is effective in mitigating the response behavior. This is consistent with the previous discussion, as the responses are dominated by waves and coverage of VIV suppression devices only affects the less important current-induced vibrations.

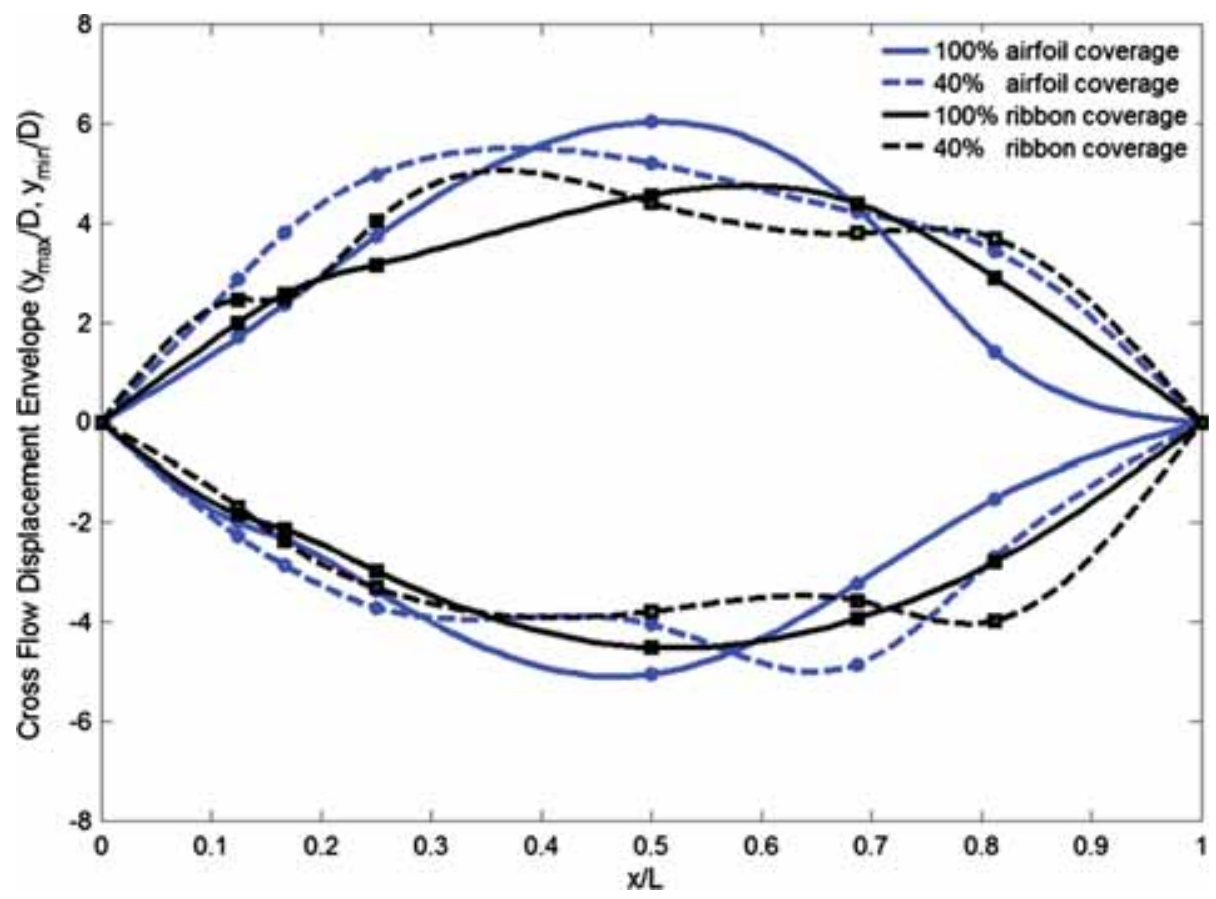

Figure 8: Response envelopes for airfoil and ribbon faired cylinders with $100 \%$ and $40 \%$ coverage. 


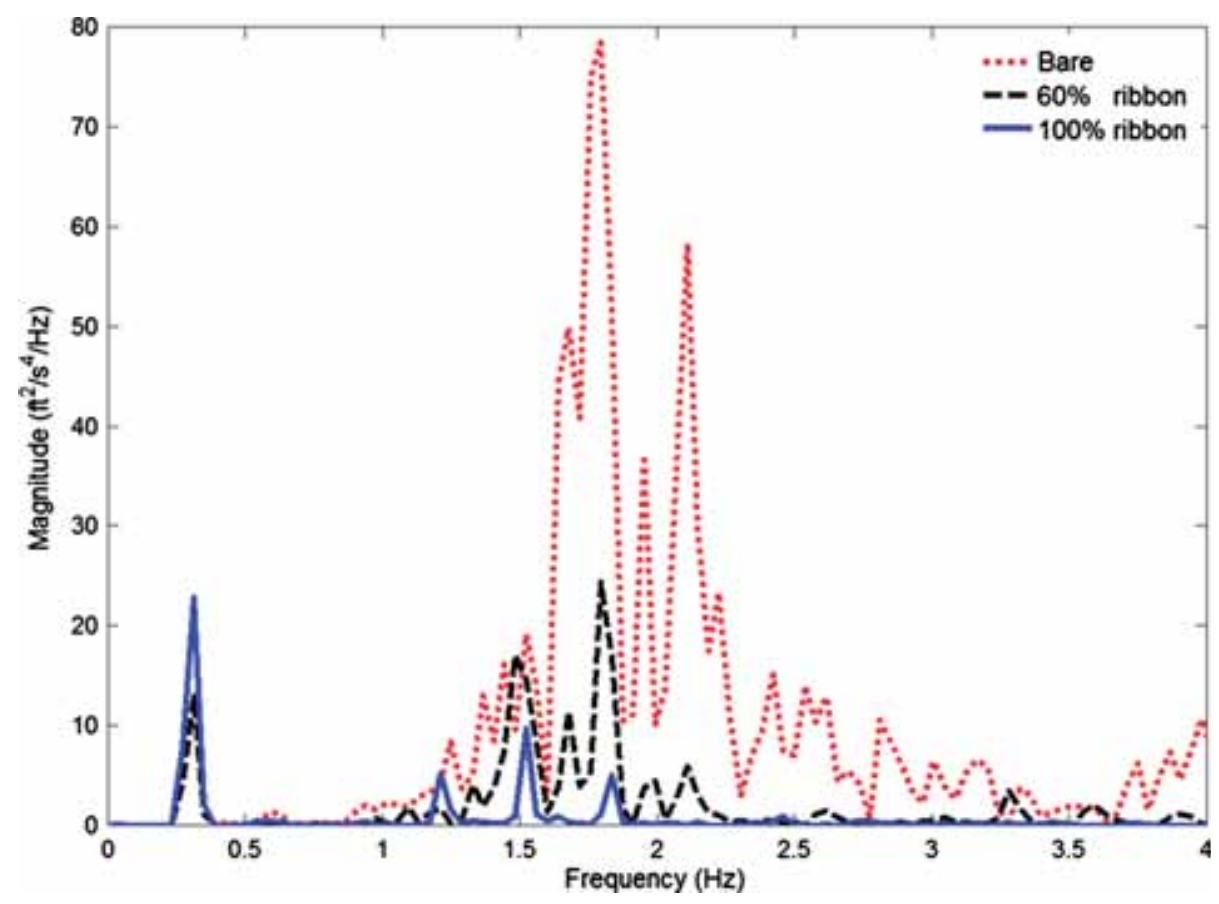

Figure 9: Power spectral densities of bare, $60 \%, 100 \%$ ribbon faired cylinders.

The modal responses of partial coverage tests in the combined loading condition further explained the phenomena that though ineffective around the wave frequency, fairings could suppress the higher order modes. For illustrative purpose, power spectral densities of bare, $60 \%$ and $100 \%$ ribbon coverage at accelerometer 4 in the middle of the cylinder are presented in Fig. 9, which showed that the higher order mode components of the vibrations were gradually suppressed by the increased coverage while the responses near the wave frequency at $0.31 \mathrm{~Hz}$ remained significant. The total energy of the responses became smaller and moved toward lower frequencies as the coverage increased. The trend of energy shifting was similar in the case of airfoil faired cylinders.

The different behaviors of the suppression devices at low and high frequencies were also reflected in the mode shapes, as shown in Fig. 10a, for the fundamental modes of 0\%, 60\% and $100 \%$ ribbon coverage and Fig. $10 \mathrm{~b}$ for the second modes of $40 \%$ airfoil and ribbon coverage. In Fig. 10a, the near symmetry of the mode shapes confirmed the insensitivities of coverage density in wave dominated responses. In Fig. 10b, both mode shapes at partial coverage were asymmetric and unbalanced, with larger values on the bare right end, which was similar to a normal 2nd mode shape, and with reduced values on the left faired end. Moreover, the reduction effect of the mode shape from the airfoil fairing seemed stronger than that from the ribbon fairing, which suggested the airfoil fairing may provide larger damping than the ribbon fairing. It was further confirmed by the estimations of the damping ratios of the excited modes, $2.3 \%$ for airfoil and $1.5 \%$ for ribbon. A possible explanation for the unbalanced mode shapes is partial coverage affected distribution of structural and added mass, as well as hydrodynamic damping across the cylinder. 


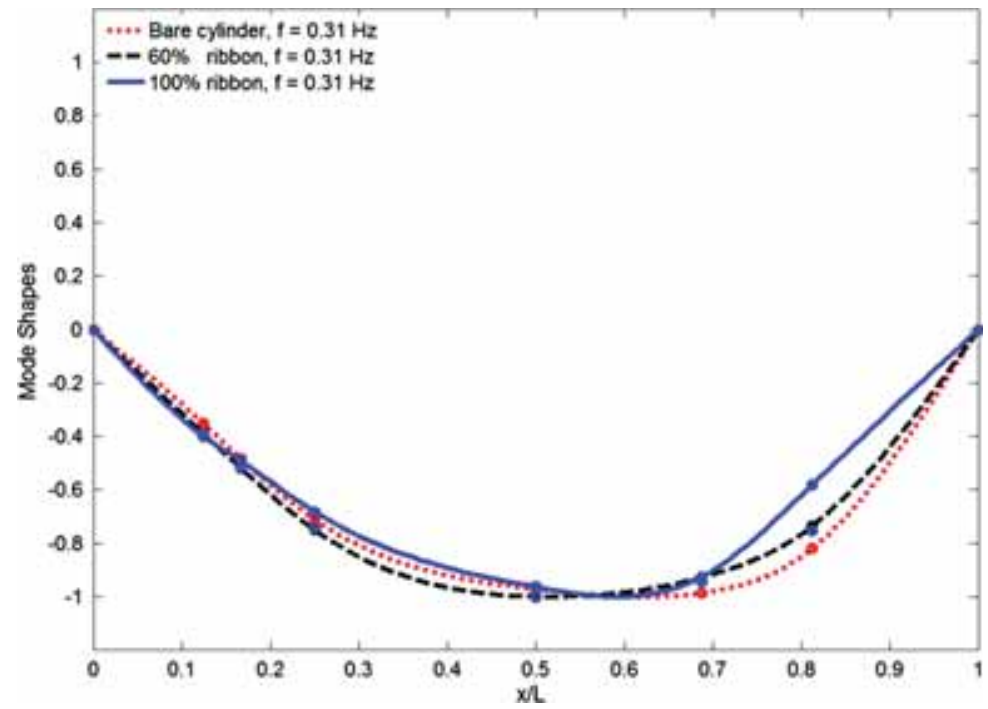

(a)

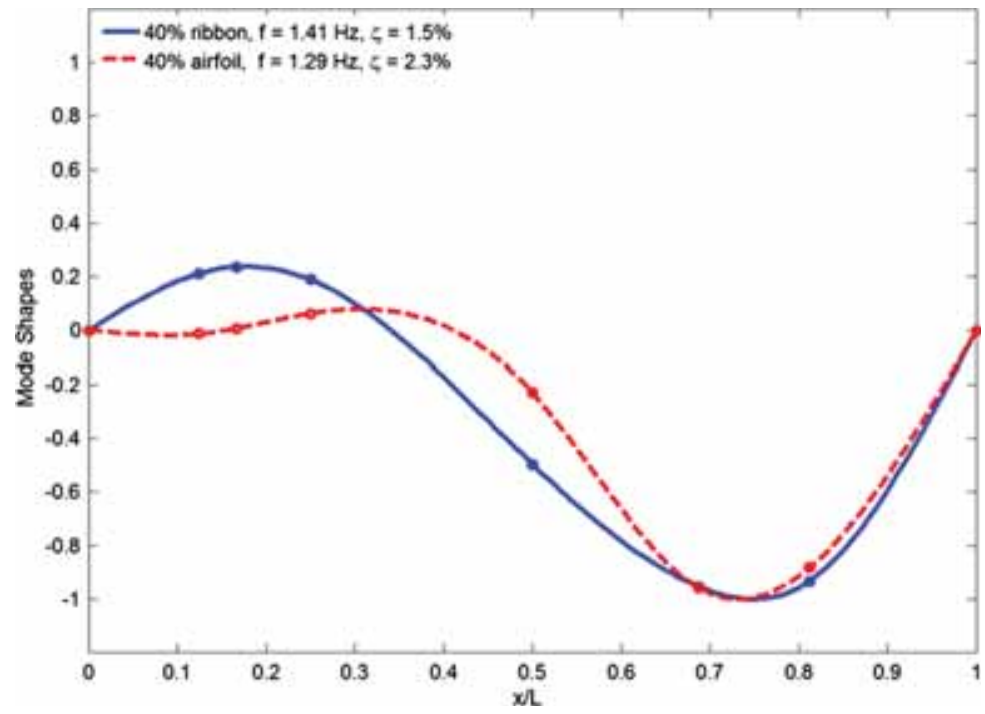

(b)

Figure 10: (a) Recovered fundamental mode shapes of bare, $60 \%$ and $100 \%$ ribbon faired cylinder. (b) Recovered unbalanced mode shape of $40 \%$ airfoil and ribbon faired cylinder test.

\section{SUMMARY}

Several features of time domain decomposition technique approach make it especially suitable for the analysis of data from a wide variety of offshore science and engineering applications, such as the analysis of flow-induced vibrations. First, the technique can be utilized to analyze measurements of system response due to ambient or controlled force excitation to resolve the modal characteristics. This is an important point as the laboratory 
experiments were conducted at relatively low sub-critical Reynolds numbers, and field data will typically be in the super-critical flow regime. Thus, the methodology presented is valid for the analysis of laboratory or field data. Secondly, the computation of single value decomposition process is only dependent on the number of sensors, but as noted the subsequent frequency resolution is important. Finally, the technique allows direct estimates of the mode shape from the measured data without making assumptions or idealizations. The current study noted the practical implementation issues regarding optimal filter selection and subsequent solutions and the need for use of the zoom technique in some cases in order to more accurately estimate the modal critical damping ratios.

The experiments reported here were conducted to investigate the effectiveness of VIV suppression performance of variable coverage of airfoil and ribbon fairings on a horizontally mounted flexible cylinder with an L/D ratio of 760 . The various cylinder configurations were towed at several different speeds to simulate uniform current velocities, and for some cases regular wave conditions were superimposed. The VIV analysis program developed for this ongoing study was employed to conduct time series analysis, modal analysis and numerical simulation, and to extract useful response information in space and modal domain. The study presented in this paper confirmed the effectiveness of both fairings in suppressing VIV in uniform current conditions only.

For the experiments where regular waves were introduced as the test cylinder was being towed, the response behavior was dominated by the orbital nature of the wave kinematics, and the functionality of both fairings types was greatly disturbed. The research findings serve to illustrate the challenges faced by offshore engineers when designing long flexible cylindrical offshore structures and flow-induced suppression devices when both currents and waves are present. Based upon the analysis of the data, it was shown that the ribbon fairings provided less average damping compared to the airfoil fairings, and that the airfoil fairings had a strong affinity to amplify the horizontal response behavior when subject to the combined current and wave conditions. It should be noted that in design practice ribbon fairings are typically used to suppress the flow-induced vibration of long vertically oriented slender cylinders or cables. The experiments also demonstrated the response behavior of test cylinder when subject to combined loads, which was not as sensitive to coverage density of fairings as might be expected, though higher modes and unbalanced mode shapes were observed in the partial coverage cases. The asymmetric response observed for the combined wave and current loading cases, which is inclusive of the bare cylinder and symmetric or asymmetric fairing coverage, might reflect the superposition effects and modal damping of the various dominant modes. Further investigation is warranted to further resolve this and other interesting observations from this study.

\section{REFERENCES}

[1] Nielsen, G.F., Soreide, T.H. \& Kvarme, S.O., VIV response of long free spanning pipelines. Proceedings of the 21st International Conference on Offshore Mechanics and Artic Engineering, OMAE 28075, 2002.

[2] Det Norske Veritas (DNV), Free Spanning Pipelines, Guideline No. 14, DNV: Høvik, Norway, 1998.

[3] Allen, D.W., Henning, D.L. \& Lee, L., Drilling riser fairing tests at prototype Reynolds numbers. Proceedings of the 26th International Conference on Offshore Mechanics and Artic Engineering, OMAE 29219, 2007.

[4] Brown, A.J. \& King, R., Tests with a flexible quasi-fairing to reduce riser drag, suppress VIV and limit drilling down-time. Offshore Technology Conference, OTC 19161, 2008. 
[5] Li, X., Bose, N., Zhu, L. \& Spencer, D., Multi-modal VIV tests for a highly flexible model riser. International Symposium on Technology of Ultra Deep Ocean Engineering, Tokyo, Japan, 2005.

[6] Prastianto, R.W., Otsuka, K. \& Ikeda, Y., A small scale experiment for multi-modal VIV response of a long flexible horizontal cylinder. Proceedings of the 17th International Offshore and Polar Engineering Conference, ISOPE, Lisbon, Portugal, 2007.

[7] Wilde, I.J.J.D. \& Huijsmans, R.H.M., Laboratory investigation of long riser VIV response. Proceedings of the 14th International Offshore and Polar Engineering Conference, ISOPE, Toulon, France, 2004.

[8] Allen, D.W. \& Henning, D.L., Fairings versus helical strakes for suppression of vortex-induced vibration: technical comparisons. Offshore Technology Conference, OTC 19373, 2008.

[9] Trim, A.D., Braaten, H., Lie, H. \& Tognarelli, M.A., Experimental investigation of vortex-induced vibration of long marine risers. Journal of Fluids and Structures, 21, 335-361, 2005. doi: http://dx.doi.org/10.1016/j.jfluidstructs.2005.07.014

[10] Niedzwecki, J.M. \& Fang, S.M., Suppression of flow-induced vibrations using ribbon fairings. Journal of Computational Methods and Experimental Measurements, in press.

[11] Every, M.J., King, R. \& Weaver, D.S., Vortex-excited vibrations of cylinders and cables and their suppression. Ocean Engineering, 9(2), 135-157, 1982. doi: http://dx.doi. org/10.1016/0029-8018(82)90010-5

[12] Kim, B.H., Stubbs, N. \& Park, T., A new method to extract modal parameters using output-only responses. Journal of Sound and Vibration, 282, 215-230, 2005. doi: http:// dx.doi.org/10.1016/j.jsv.2004.02.026

[13] Chitwood, J.S., Vortex-induced vibration of a slender horizontal cylinder in currents and waves. OTRCreport No. 2/98-A9575, 1998.

[14] Chung, T.Y., Vortex-induced vibration of flexible cylinders in sheared flows. Ph.D. dissertation, MIT Department of Ocean Engineering, Cambridge, Mass, USA, 1987.

[15] Bendat, J.S. \& Piersol, A.G., Engineering Applications of Correlation and Spectral Analysis, Wiley: New York, 1980.

[16] Randall, R.B., Frequency Analysis, Bruel \& Kjaer: Denmark, 1987.

[17] Niedzwecki, J.M. \& Liagre, P.-Y.F., System identification of distributed-parameter marine riser models. Ocean Engineering, 30, 1387-1415, 2003. doi: http://dx.doi. org/10.1016/S0029-8018(02)00110-5 